\title{
ОСНОВНЫЕ ФОРМУЛЫ ТЕОРИИ ИНТЕРФЕРЕНЦИОННО-ПОЛЯРИЗАЦИОННЫХ СВЕТОФИЛЬТРОВ
}

\section{Введение}

В [ ${ }^{1}$ ] была развита по образцу теории многослойных интерференционных пленок теория интерференционно-поляризационных светофильтров типа Шольца, а в $\left[{ }^{2}\right]$ было показано применение этой теории к синтезу таких фильтров. В настоящей статье нашей целью является, во-первых, приведение основных формул теории к более простому виду и, во-вторых, вывод некоторых дальнейших следствий, полезных при более детальном исследовании свойств светофильтров.

Напомним, что фильтр типа Шольца состоит из последовательности параллельно расположенных одна за другой плоскопараллельных двупреломляющих пластинок, оптические оси которых параллельны их поверхностям. Эта стопа пластинок обрамлена входным и выходным поляризаторами. Свет проходит фильтр обычно в перпендикулярном пластинкам направлении, принимаемом за ось $z$. Каждая пластинка характеризуется толщиной $h$ и азимутом $\varphi$, т. е. углом, образуемым оптической осью с произвольно выбранной осью $x$. Эллиптическую поляризацию идущей в направлении оси $z$ монохроматической световой волны описываем матрицей

$$
A=\left(\begin{array}{l}
A_{x} \\
A_{y}
\end{array}\right)
$$

где $A_{x}, A_{y}-$ компоненты комплексной амплитуды электрического вектора волны. Через $L$ обозначаем матрицу, согласно которой матрица поляризации преобразуется при прохождении волны сквозь одну пластинку. Тогда, если $N$ - число пластинок, то матрица $A^{\prime}$ прошедшего сквозь все пластинки света связана с матрицей падающего на первую пластинку света формулой

$$
A^{\prime}=F A \text {, }
$$

где

$$
F=L_{N} L_{N-1} \ldots L_{1} .
$$

Здесь, как и ранее, мы пренебрегаем отражением света на поверхностях пластинок.

Для матриц $L_{k}$ имеем формулу

$$
L_{k}=\Phi\left(-\varphi_{k}\right) \Gamma\left(\gamma_{k}\right) \Phi\left(\varphi_{k}\right)
$$

или, равносильно,

$$
L_{k}=\cos \gamma_{k} E+i \sin \gamma_{k} \bar{\Phi}\left(2 \varphi_{k}\right) \text {. }
$$


В этих формулах $E-$ единичная матрица,

$$
\begin{aligned}
& \Phi(\varphi)=\left(\begin{array}{rr}
\cos \varphi & \sin \varphi \\
-\sin \varphi & \cos \varphi
\end{array}\right), \\
& \bar{\Phi}(\varphi)=\left(\begin{array}{lr}
\cos \varphi & \sin \varphi \\
\sin \varphi & -\cos \varphi
\end{array}\right)
\end{aligned}
$$

и

$$
\Gamma(\gamma)=\left(\begin{array}{cc}
e^{i \gamma} & 0 \\
0 & e^{-i \gamma}
\end{array}\right)
$$

Определим вдобавок матрицу

$$
\bar{\Gamma}(\gamma)=\left(\begin{array}{cc}
0 & i e^{-i \gamma} \\
-i e^{i \gamma} & 0
\end{array}\right) .
$$

Аргумент $\gamma_{k}$ определяется формулой

$$
\gamma_{k^{\prime}}=\left(\pi h_{k} / \lambda\right)\left(n_{o}-n_{e}\right),
$$

где $\lambda$ - длина волны, $n_{o}-$ обыкновенный и $n_{e}-$ необыкновенный показатель преломления пластинок.

Матрицы $\Phi$ и $\bar{\Phi}$, а также $\Gamma$ и $\bar{\Gamma}$, удовлетворяют соотношениям одинакового вида

$$
\begin{aligned}
& \Phi\left(\varphi_{2}\right) \Phi\left(\varphi_{1}\right)=\Phi\left(\varphi_{1}+\varphi_{2}\right), \\
& \bar{\Phi}\left(\varphi_{2}\right) \bar{\Phi}\left(\varphi_{1}\right)=\Phi\left(\varphi_{1}-\varphi_{2}\right), \\
& \bar{\Phi}\left(\varphi_{2}\right) \Phi\left(\varphi_{1}\right)=\bar{\Phi}\left(\varphi_{1}+\varphi_{2}\right), \\
& \Phi\left(\varphi_{2}\right) \bar{\Phi}\left(\varphi_{1}\right)=\bar{\Phi}\left(\varphi_{1}-\varphi_{2}\right)
\end{aligned}
$$

и

$$
\begin{aligned}
& \Gamma\left(\gamma_{2}\right) \Gamma\left(\gamma_{1}\right)=\Gamma\left(\gamma_{1}+\gamma_{2}\right), \\
& \bar{\Gamma}\left(\gamma_{2}\right) \bar{\Gamma}\left(\gamma_{1}\right)=\Gamma\left(\gamma_{1}-\gamma_{2}\right), \\
& \bar{\Gamma}\left(\gamma_{2}\right) \Gamma\left(\gamma_{1}\right)=\bar{\Gamma}\left(\gamma_{1}+\gamma_{2}\right), \\
& \Gamma\left(\gamma_{2}\right) \bar{\Gamma}\left(\gamma_{1}\right)=\bar{\Gamma}\left(\gamma_{1}-\gamma_{2}\right) .
\end{aligned}
$$

Отметим, что матрица $Ф(\varphi)$ означает у нас теперь то же, что матрица $\Phi(-\varphi)$ в $\left[{ }^{1}\right]$, матрица $\Gamma(\gamma)$ то же, что $M(\gamma)$, а матрица $\bar{\Gamma}(\gamma)$ то же, что

$$
\left(\begin{array}{rr}
0 & 1 \\
-1 & 0
\end{array}\right) \bar{M}(\gamma)\left(\begin{array}{ll}
0 & i \\
i & 0
\end{array}\right)
$$

Смысл матрицы $\bar{\Phi}(\varphi)$ остался прежним.

\section{Основные формулы}

Для матрицы $F$, преобразующей, согласно формуле (2), матрицу поляризации пропускаемого стопой пластинок света, можно найти две простые формулы. Первая получается прямым перемножением в формуле (3) матриц $L_{k}$, взятых в виде (5). С учетом второй и третьей формул (11) получаем следующее выражение

$$
F=\Sigma i^{s} \sin \gamma_{k_{1}} \sin \gamma_{k_{2}} \ldots \sin \gamma_{k_{s}} \cos \gamma_{l_{1}} \cos \gamma_{l_{2}} \ldots \cos \gamma_{l_{N-s}} \times
$$




$$
{\stackrel{(-)^{s}}{\Phi}}^{s}\left(2 \varphi_{k_{1}}-2 \varphi_{k_{2}}+-\ldots-(-1)^{s} 2 \varphi_{k_{s}}\right),
$$

где сумма берется по всем разбиениям индексов $1,2, \ldots, N$ на две группы: $k_{1}, k_{2}, \ldots, k_{s}$ и $l_{1}, l_{2}, \ldots, l_{N-s}$, причем $k_{1}<k_{2}<\ldots<k_{s}$ и $s=0,1, \ldots, N$. В сумме, очевидно, $2^{N}$ членов. ${\stackrel{(-)^{s}}{\Phi}}^{s}$ означает Ф при $s$ четном и $\bar{\Phi}$ при $s$ нечетном. Для элементов матрицы $F$ имеем, согласно (6) и (7), формулы

$$
\begin{aligned}
F_{11}=F_{22}^{*}= & \sum i^{s} \sin \gamma_{k_{1}} \sin \gamma_{k_{2}} \ldots \sin \gamma_{k_{s}} \cos \gamma_{l_{1}} \cos \gamma_{l_{2}} \ldots \cos \gamma_{l_{N-s}} X \\
& \times \cos \left(2 \varphi_{k_{1}}-2 \varphi_{k_{2}}+\ldots \ldots-(-1)^{s} 2 \varphi_{k_{s}}\right)
\end{aligned}
$$

и

$$
\begin{aligned}
F_{12}=-F_{21}^{*}= & \sum i^{s} \sin \gamma_{k_{1}} \sin \gamma_{k_{2}} \ldots \sin \gamma_{k_{s}} \cos \gamma_{l_{1}} \cos \gamma_{l_{2}} \ldots \cos \gamma_{l_{N-s}} \times \\
& \times \sin \left(2 \varphi_{k_{1}}-2 \varphi_{k_{2}}+-\ldots-(-1)^{s} 2 \varphi_{k_{s}}\right) .
\end{aligned}
$$

Другая формула для $F$ получается следующим образом. Подставляя в формулу (3) выражения (4) для $L_{k}$, находим

$$
\begin{gathered}
F=\Phi\left(-\varphi_{N}\right) \Gamma\left(\gamma_{N}\right) \Phi\left(\varphi_{N}\right) \Phi\left(-\varphi_{N-1}\right) \Gamma\left(\gamma_{N-1}\right) \Phi\left(\varphi_{N-1}\right) \ldots \\
\ldots \Phi\left(-\varphi_{2}\right) \Gamma\left(\gamma_{2}\right) \Phi\left(\varphi_{2}\right) \Phi\left(-\varphi_{1}\right) \Gamma\left(\gamma_{1}\right) \Phi\left(\varphi_{1}\right) .
\end{gathered}
$$

Обозначив

так что

$$
\varphi_{0}=\varphi_{N+1} \equiv 0,
$$

$$
\Phi\left(-\varphi_{0}\right):=\Phi\left(\varphi_{N+1}\right)=E \text {, }
$$

и

$$
\varphi_{k, k+1}=\varphi_{k}-\varphi_{k+1}, \quad k=0,1, \ldots, N,
$$

перепишем, учитывая первую формулу (11), выражение (16) в виде

$$
\begin{gathered}
F=\Phi\left(-\varphi_{N, N+1}\right) \Gamma\left(\gamma_{N}\right) \Phi\left(-\varphi_{N-1, N}\right) \Gamma\left(\gamma_{N-1}\right) \ldots \\
\ldots \Phi\left(-\varphi_{23}\right) \Gamma\left(\gamma_{2}\right) \Phi\left(-\varphi_{12}\right) \Gamma\left(\gamma_{1}\right) \Phi\left(-\varphi_{01}\right) .
\end{gathered}
$$

Учитывая первую формулу (12), находим

$$
\begin{gathered}
F=\Gamma\left(\gamma_{0 N}\right)\left[\Gamma\left(-\gamma_{0 N}\right) \Phi\left(-\varphi_{N, N+1}\right) \Gamma\left(\gamma_{0 N}\right)\right] \times \\
\times\left[\Gamma\left(-\gamma_{0, N-1}\right) \Phi\left(-\varphi_{N-1, N}\right) \Gamma\left(\gamma_{0, N-1}\right)\right] \ldots \\
\ldots\left[\Gamma\left(-\gamma_{01}\right) \Phi\left(-\varphi_{12}\right) \Gamma\left(\gamma_{01}\right)\right]\left[\Gamma\left(-\gamma_{00}\right) \Phi\left(-\varphi_{01}\right) \Gamma\left(\gamma_{00}\right)\right],
\end{gathered}
$$

где

Обозначим

$$
\begin{gathered}
\gamma_{0}=\gamma_{00}=0, \\
\gamma_{0 k}=\gamma_{0}+\gamma_{1}+\ldots+\gamma_{k}, \quad k=0,1, \ldots, N .
\end{gathered}
$$

тогда

$$
Q_{k}=\Gamma\left(-\gamma_{0 k}\right) \Phi\left(-\varphi_{k, k+1}\right) \Gamma\left(\gamma_{0 k}\right)
$$

$$
F=\Gamma\left(\gamma_{0 N}\right) Q_{N} Q_{N-1} \ldots Q_{1} Q_{0} .
$$

Перемножая матрицы в формуле (23), находим другое выражение для $Q_{k}$ :

$$
Q_{k}=\cos \varphi_{k, k+1} E+i \sin \varphi_{k, k+1} \bar{\Gamma}\left(2 \gamma_{0 k}\right) .
$$

Формулы (23) и (25) аналогичны формулам (4) и (5), а формула (24) - формуле (3). 
Остается перемножить матрицы в формуле (24), беря все $Q$ в виде (25). В результате, учитывая формулы (12), приходим к аналогичному (13) выражению

$$
F=\Sigma i^{s} \times \varphi_{01} \times \varphi_{12} \ldots \times \varphi_{N, N+1} \stackrel{(-)}{\Gamma}^{8}\left(\varepsilon_{1} \gamma_{1}+\varepsilon_{2} \gamma_{2}+\ldots+\varepsilon_{N} \gamma_{N}\right),
$$

где $\mathrm{x} \varphi$ означает $\sin \varphi$ или $\cos \varphi$, причем сумма берется по всем $2^{N+1}$ распределениям х на синусы и косинусы, а $s$ означает число синусов $(s=0,1, \ldots, N+1) ; \stackrel{(-)^{s}}{\Gamma}$ означает $\Gamma$ при $s$ четном и $\bar{\Gamma}$ при $s$ нечетном; $\varepsilon_{1}, \varepsilon_{2}, \ldots, \varepsilon_{N}$ равны +1 или -1 согласно правилу: $\varepsilon_{k} \varepsilon_{k+1}= \pm 1$, если $\left.\quad \varphi_{k, k+1}=\begin{array}{c}\cos \\ \sin \end{array}\right\} \varphi_{k, k+1}$, причем $\varepsilon_{0}=1$. Отсюда ясно, что множитель х $\varphi_{N, N+1}$ не участвует в определении знаков членов аргумента $\stackrel{(-)^{s}}{\Gamma}$. Он может быть или синусом, или косинусом, и в обоих случаях аргумент $\stackrel{(-)^{s}}{\Gamma}$ один и тот же, но в одном случае $\stackrel{(-)^{8}}{\Gamma}$ есть $Г$, а в другом $\bar{\Gamma}$.

В силу формул (8) и (9) из формулы (26) находим следующие выражения матричных элементов:

$$
F_{11}=F_{22}^{*}=\sum_{s \text { четно }} i^{s} \mathrm{X} \varphi_{01} \times \varphi_{12} \ldots \times \varphi_{N, N+1} \exp \left[i\left(\varepsilon_{1} \gamma_{1}+\varepsilon_{2} \gamma_{2}+\ldots+\varepsilon_{N} \gamma_{N}\right)\right]
$$

H

$$
F_{21}=-F_{12}^{*}=\sum_{s \text { нечетно }} i^{s-1} \mathrm{x} \varphi_{01} \mathrm{X}_{12} \ldots \mathrm{x} \varphi_{N, N+1} \exp \left[i\left(\varepsilon_{1} \gamma_{1}+\varepsilon_{2} \gamma_{2}+\ldots+\varepsilon_{N} \gamma_{N}\right)\right] .
$$

Выведенные в этом разделе основные формулы сходны с полученными в $\left[{ }^{1}\right]$, но проще и удобнее тех.

\section{Разложение в ряд коэффициента пропускания фильтра}

Примем направление входного поляризатора за ось $x$ и амплитуду прошедшего поляризатор света за единицу, так что

$$
A=\left(\begin{array}{l}
1 \\
0
\end{array}\right) \text {. }
$$

Тогда

$$
A^{\prime}=\left(\begin{array}{l}
F_{11} \\
F_{21}
\end{array}\right)
$$

т. е. амплитуда прошедшего фильтр света равна $F_{11}$, если выходной поляризатор параллелен входному, и $F_{21}$, если выходной поляризатор скрещен с входным. Коэффициент пропускания фильтра равен в первом случае $F_{11} F_{11}{ }^{*}$, а во втором $F_{21} F_{21}{ }^{*}$. А так как, согласно формулам (14) и (15), выражения $F_{11}$ и $F_{21}$ сходны, и притом, в силу формул (3) и (4),

$$
F_{11} F_{22}-F_{12} F_{2 \neq}=F_{11} F_{11}^{*}+F_{21} F_{21}^{*}=1,
$$

то достаточно рассмотреть подробно только один из коэффициентов, например, $F_{11} F_{11}{ }^{*}$.

В этом разделе, продолжая аналогию с теорией интерференцион- 
ных пленок (см. [ $\left.\left.{ }^{3}\right], \S 12\right)$, разложим коэффициент пропускания фильтра в ряд. Для этого есть два способа, но в настоящей статье изложим только один из них. Разложение позволит вывести для коэффициента пропускания очень простую приближенную формулу.

Исходим из формулы (14). Перепишем ее, введя для удобства величины $\varphi_{0}$ и $\varphi_{N+1}$ (см. формулу (17)), в виде

$$
\begin{gathered}
F_{11}=\sum i^{s} \sin \gamma_{k_{1}} \sin \gamma_{k_{2}} \ldots \sin \gamma_{k_{s}} \cos \gamma_{l_{1}} \cos \gamma_{l_{2}} \ldots \cos \gamma_{l_{N-s}} \times \\
\times \cos \left(\varphi_{0}-2 \varphi_{k_{1}}+2 \varphi_{k_{2}}-+\ldots+(-1)^{s} 2 \varphi_{k_{s}}-(-1)^{s} \varphi_{N+1}\right) .
\end{gathered}
$$

Рассматривая $F_{11}$ как функцию $\gamma_{1}, \gamma_{2}, \ldots, \gamma_{N}$, выразим эти аргументы в виде

$$
\gamma_{k}=g_{k} \pi / 2+\Delta \gamma_{k}, \quad k=1,2, \ldots, N,
$$

где $g_{k}$ - целые числа, а $\Delta \gamma_{k}$ возможно малы, и будем разлагать $F_{11} F_{11}{ }^{*}$ в ряд Тейлора по степеням $\Delta \gamma_{k}$. Для этого найдем сначала значение $F_{11}$ и производных этой величины при всех $\Delta \gamma_{k}=0$.

Пусть $N_{r}$ есть число пластинок, у которых

$$
g_{k} \equiv r(\bmod 4), \quad r=1,2,3,4 .
$$

Положив в формуле (32) все $\Delta \gamma_{k}=0$, видим, что в сумме все члены, кроме одного, обращаются в нуль. Отличным от нуля оказывается тот член, в котором аргументами синусов являются все $\gamma_{k}$ с нечетным $r$, а аргументами косинусов все $\gamma_{k}$ с четным $r$. Следовательно,

$$
\left(F_{11}\right)_{0}=i^{N_{1}+N_{3}}(-1)^{N_{2}+N_{3}} \cos \theta_{0, N+1},
$$

где

$$
\theta_{0, N+1}=\varphi_{0}-2 \varphi m_{1}+2 \varphi m_{2}-+\ldots+(-1)^{N_{1}+N_{3}} 2 \varphi_{m_{N_{1}+N_{3}}}-(-1)^{N_{1}+N_{3}} \varphi_{N+1},
$$

причем $m_{1}<m_{2}<\ldots<m_{N_{1}+N_{3}}$ и эти индексы принадлежат пластинкам с нечетными $r$.

Выразим далее производные от $F_{11}$ по $\gamma_{k}$, взятые при всех $\Delta \gamma_{k}=0$. Так как производная от синуса равна косинусу, а от косинуса оинусу с минусом, то единственный член $F_{11}$, производная которого по $\gamma_{k}$ отлична при всех $\Delta \gamma_{k}=0$ от нуля, содержит $\cos \gamma_{k}$ или $\sin \gamma_{k}$, если $r$ нечетно или четно, соответственно. Следовательно, для производной получится сходное с формулой (35) выражение, только добавляется множитель $i$ и вместо $\theta_{0, N+1}$ аргументом косинуса будет аналогичное (36) выражение, но без члена $\pm 2 \varphi_{k}$, если он входит в $\theta_{0, N+1}$, и с этим членом, если его в $\theta_{0, N+1}$ нет. Согласно сказанному пишем формулу

$$
\left(F_{11, k}\right)_{0}=i^{N_{1}+N_{3}+1}(-1)^{N_{2}+N_{3}} \cos \left(\theta_{0 k}-\theta_{k, N+1}\right),
$$

где индекс $k$ у $F_{11}$ означает взятую по $\gamma_{k}$ производную, а $\theta_{0 k}$ и $\theta_{k, N+1}$ определяются следующим образом. $\theta_{0 k}$ содержит все те члены из $\theta_{0, N+1}$, индексы которых меньше $k$, а также член $\varphi_{k}$ (независимо от наличия или отсутствия члена $2 \varphi_{k}$ в $\theta_{0, N+1}$ ) со знаком, противоположным знаку предшествующего ему члена; $\theta_{k, N+1}$ содержит все те члены из $\theta_{0, N+1}$, индексы которых больше $k$, а также член $\varphi_{k}$ (независимо от наличия или отсутствия члена $2 \varphi_{k}$ в $\left.\theta_{0, N+1}\right)$ со знаком, противоположным знаку следующего за ним члена.

Рассуждая таким же образом и дальше, находим формулу второй производной

$$
\left(F_{11, k l}\right)_{0}=i^{N_{1}+N_{3}+2}(-1)^{N_{2}+N_{3}} \cos \left(\theta_{0 k}-\theta_{k l}+\theta_{l, N+1}\right), \quad k \leqslant l,
$$


где $\theta_{k l}$ определяются формулой

$$
\theta_{k l}=\theta_{0, N+1}-\theta_{0 k}-\theta_{l, N+1} .
$$

Легкс видеть, что

$$
\theta_{k l}+\theta_{l m}=\theta_{k m},
$$

где $0 \leqslant k \leqslant l \leqslant m \leqslant N+1$, и

$$
\theta_{k k}=0 .
$$

Наконец, общая формула производной $n$-го порядка (при всех $\Delta \gamma_{k}=0$ ) получается в виде

$$
\begin{gathered}
\left(F_{11, k_{1} k_{2} \ldots k_{n}}\right)_{0}=i^{N_{1}+N_{3}+n}(-1)^{N_{2}+N_{3}} \times \\
\times \cos \left(\theta_{0 k_{1}}-\theta_{k_{1} k_{2},}+-\ldots-(-1)^{n} \theta_{k_{n-1} k_{n}}+(-1)^{n} \theta_{k_{n}, N+1}\right), \\
k_{1} \leqslant k_{2} \leqslant \ldots \leqslant k_{n} .
\end{gathered}
$$

Далее выразим производные от $F_{11} F_{11}{ }^{*}$. Так как эта величина является четной функцией от всех $\gamma_{k}$, то все производные нечетного порядка равны нулю. А общая формула производной четного порядка $2 n$ имеет вид

$$
\left(F_{11} F_{11}^{*}\right)_{k_{1} k_{2} \ldots k_{2 n}}=\sum F_{11, l_{1} l_{2} \ldots l_{p}} F_{11, m_{1} m_{2} \ldots m_{2 n-p}}^{*},
$$

где $\quad k_{1} \leqslant k_{2} \leqslant \ldots \leqslant k_{2 n}, \quad l_{1} \leqslant l_{2} \leqslant \ldots \leqslant l_{p}, \quad m_{1} \leqslant m_{2} \leqslant \ldots \leqslant m_{2 n-p}$; совокупность индексов $k$ тождественна совокупности индексов $l$ и $m$, а сумма берется по всем $2^{2 n}$ комбинациям разбиения совокупности индексов $k$ на совокупности индексов $l$ и $m$. Для простоты здесь и далее опускается индекс 0 , означающий, что производные берутся при всех $\Delta \gamma_{k}=0$. Подставляя в правую часть формулы (43) выражения производных из формулы (42), находим

$$
\begin{gathered}
\left(F_{11} F_{11}^{*}\right)_{k_{1} k_{2} \ldots k_{2 n}}= \\
=\Sigma(-1)^{n+p} \cos \left(\theta_{0 l_{1}}-\theta_{l_{1} l_{2}}+-\ldots-(-1)^{p} \theta_{l_{p-1} l_{p}}+(-1)^{p} \theta_{l_{p}, N+1}\right) \times \\
\times \cos \left(\theta_{0 m_{1}}-\theta_{m_{1} m_{2}}+-\ldots-(-1)^{p} \theta_{m_{2 n-p-1} m_{2 n-p}}+(-1)^{p} \theta_{m_{2 n-p}, N+1},\right.
\end{gathered}
$$

причем члены в сумме, очевидно, попарно равны. Для упрощения этой формулы обратим внимание на то, что аргументы всех косинусов можно написать в виде

$$
\theta_{0 k_{1}} \pm \theta_{k_{1} k_{2}} \pm \ldots \pm \theta_{k_{2 n-1} k_{2 n}}+(-1)^{p} \theta_{k_{2 n}, N+1}
$$

(см. формулу (40)), причем каждая из $2^{2 n-1}$ комбинаций знаков членов написанных в этом виде аргументов повторяется в формуле дважды, а в одном и том же члене суммы каждому сохранению знака в последовательности знаков одного из аргументов соответствует перемена знака в другом, и наоборот. Поэтому, преобразуя произведение косинусов в половину суммы косинусов от суммы и разности аргументов, находим

$$
\begin{gathered}
\left(F_{11} F_{11}\right)_{k_{1} k_{2} \ldots k_{2 n}}=2^{n-1} \Sigma(-1)^{n+p} \cos \left(2 \theta_{0 k_{1}} \pm 2 \theta_{k_{2} k_{3}} \pm \ldots \pm 2 \theta_{k_{2 n}, N+1}\right)+ \\
+2^{n-1} \Sigma(-1)^{n+p} \cos \left( \pm 2 \theta_{k_{1} k_{2}} \pm 2 \theta_{k_{3} k_{4}} \pm \ldots \pm 2 \theta_{k_{2 n-1} k_{2 n}}\right),
\end{gathered}
$$

где обе суммы берутся по всем $2^{n}$ комбинациям знаков членов аргумента косинуса. Легко видеть, что во второй сумме все члены попарно сокращаются, так как члены, различающиеся знаками всех членов 
аргумента косинуса, различаются четностью $p$. Наоборот, в первой сумме четность $p$ влияет только на знак последнего члена аргумента косинуса, так что формула (45) принимает вид

$$
\begin{gathered}
\left(F_{11} F_{11}^{* *}\right)_{k_{1} k_{2} \ldots k_{2 n}}=2^{n-1}(-1)^{n} \sum\left\{\operatorname { c o s } \left(2 \theta_{0 k_{1}} \pm 2 \theta_{k_{2} k_{3}} \pm \ldots \pm 2 \theta_{k_{2 n-2} k_{2 n-1}}+\right.\right. \\
\left.\left.+2 \theta_{k_{2 n}, N+1}\right)-\cos \left(2 \theta_{0 k_{1}} \pm 2 \theta_{k_{2} k_{3}} \pm \ldots \pm 2 \theta_{k_{2 n-2} k_{2 n-1}}-2 \theta_{k_{2 n}, N+1}\right)\right\} .
\end{gathered}
$$

Сумма берется теперь по $2^{n-1}$ комбинациям знаков. Преобразуя разность косинусов в удвоенное произведение синусов полусуммы и полуразности, находим

$$
\begin{gathered}
\left(F_{11} F_{11}^{*}\right)_{k_{1} k_{2} \ldots k_{2 n}}=-2^{n}(-1)^{n} \sum \sin \left(2 \theta_{0 k_{1}} \pm 2 \theta_{k_{2} k_{3}} \pm \ldots\right. \\
\left.\ldots \pm 2 \theta_{k_{2 n-2} k_{2 n-1}}\right) \sin 2 \theta_{k_{2 n}, N+1} .
\end{gathered}
$$

Отсюда окончательно

$$
\begin{gathered}
\left(F_{11} F_{11}^{*}\right)_{h_{1} k_{2} \ldots k_{2 n}}=(-1)^{n+1} 2^{2 n-1} \sin 2 \theta_{0 k_{1}} \cos 2 \theta_{k_{2} k_{3}} \ldots \\
\ldots \cos 2 \theta_{k_{n-2} k_{2 n-1}} \sin 2 \theta_{k_{2 n}, N+1} \\
k_{1} \leqslant k_{2} \leqslant \ldots \leqslant k_{2 n} .
\end{gathered}
$$

Теперь мы можем написать ряд Тейлора для $F_{11} F_{11}{ }^{*}$. Учитывая формулы (35) и (48), находим

$$
F_{11} F_{11}^{*}=\cos ^{2} \theta_{0, N+1}-\sum_{n=1}^{\infty}(-1)^{n} \frac{2^{2 n-1}}{(2 n) !_{1 \leqslant k_{1} \leqslant k_{2} \leqslant \ldots \leqslant k_{2 n} \leqslant N}} \sum_{k_{1} k_{2} \ldots k_{2 n}} \times
$$

$$
X \sin 2 \theta_{0 k_{1}} \cos 2 \theta_{k_{2} k_{3}} \ldots \cos 2 \theta_{k_{2 n-2} k_{2 n-1}} \sin 2 \theta_{k_{2 n}, N+1} \Delta \gamma_{k_{1}} \Delta \gamma_{k_{2}} \ldots \Delta \gamma_{k_{2 n}},
$$

где $P$ означает число перестановок индексов.

Совершенно аналогичным образом можно получить разложение в ряд Тейлора и для коэффициента $F_{21} F_{21}{ }^{*}$. Проще, однако, воспользоваться формулой (31), которая сразу дает

$$
\begin{aligned}
& F_{21} F_{21}^{*}=\sin ^{2} \theta_{0, N+1}+\sum_{n=1}^{\infty}(-1)^{n} \frac{2^{2 n-1}}{(2 n) !} \sum_{1 \leqslant k_{1} \leqslant k_{2} \leqslant \ldots \leqslant k_{2 n} \leqslant N} P_{k_{1} k_{2} \ldots k_{2 n}} X \\
& \times \sin 2 \theta_{0 k_{1}} \cos 2 \theta_{k_{2} k_{3}} \ldots \cos 2 \theta_{k_{2 n-2} k_{2 n-1}} \sin 2 \theta_{k_{2 n}, N+1} \Delta \gamma_{k_{1}} \Delta \gamma_{k_{2}} \ldots \Delta \gamma_{k_{2 n}} .
\end{aligned}
$$

\section{Приближенная формула коэффициента пропускания узкополосного фильтра}

При выводе разложений (49) и (50) мы не делали никаких предположений о составе фильтра, т. е. о толщинах и азимутах пластинок. В этом смысле эти разложения являются вполне общими. Применим их теперь к узкополосному фильтру, подобному синтезированным в [ $\left.{ }^{2}\right]$. Примем толщины пластинок равными, причем при всех $\Delta \gamma_{k}=0$, т. е. в середине полосы пропускания фильтра, коэффициент пропускания должен быть равен единице. Это значит, что в случае параллельно ориентированных входного и выходного поляризаторов должно быть

$$
\cos ^{2} \theta_{0, N+1}=1 \text {, }
$$

а в случае скрещенных поляризаторов

$$
\sin ^{2} \theta_{0, N+1}=1 \text {. }
$$


Отсюда следует, что $\sin 2 \theta_{0, N+1}=0$ и $\cos 2 \theta_{0, N+1}= \pm 1$, где верхний знак соответствует первому, а·нижний - второму случаю. Следовательно, согласно формуле (40),

$$
\sin 2 \theta_{l, N+1}=\mp \sin 2 \theta_{0 l} \text {. }
$$

Кроме того, сделаем во всех членах формул (49) и (50), начиная с членов четвертого порядка (т. е. при $n \geqslant 2$ ), приближенную подстановку

$\cos 2 \theta_{l m}=\cos 2 \theta_{0 l} \cos 2 \theta_{0 m}+\sin 2 \theta_{0 l} \sin 2 \theta_{0 m} \approx \sin 2 \theta_{0 l} \sin 2 \theta_{0 m}$.

Обе формулы примут тогда одинаковый вид. Именно, для $F_{11} F_{11}{ }^{*}$ имеем:

$$
F_{11} F_{11}^{*} \approx \frac{1}{2}+\sum_{n=0}^{\infty} \frac{(-1)^{n} 2^{2 n-1}}{(2 n) !}\left(\sum_{k=1}^{N} \sin 2 \theta_{0 k} \Delta \gamma_{k}\right)^{2 n} .
$$

Этот ряд является разложением квадрата косинуса, так что окончательно

$$
F_{11} F_{11}^{*} \approx \cos ^{2}\left(\sum_{k=1}^{N} \sin 2 \theta_{0 k} \Delta \gamma_{k}\right)
$$

Для $F_{21} F_{21}{ }^{*}$ получается совершенно такая же формула, только, конечно, в ней величины $2 \theta_{0 k}$ будут иные в соответствии с различием формул (51) и $(52)$.

Формула (56) является нашим главным результатом. Она может найти двоякое применение. Во-первых, она дает приближенный ход спектральной кривой пропускания фильтра. Так как все пластинки фильтра типа Шольца имеют одинаковую толщину, то все $\Delta \gamma_{k}$ равны. Примером могут послужить два синтезированных в $\left[{ }^{2}\right]$ фильтра. Для первого из них (c $f=1 / 3$ ) приближенная формула получается в виде (одинаковом во всех четырех вариантах)

$$
F_{21} F_{21}^{*}=\cos ^{2}(4,140 \Delta \gamma)
$$

а для второго (с $f=0,4)$ - в виде

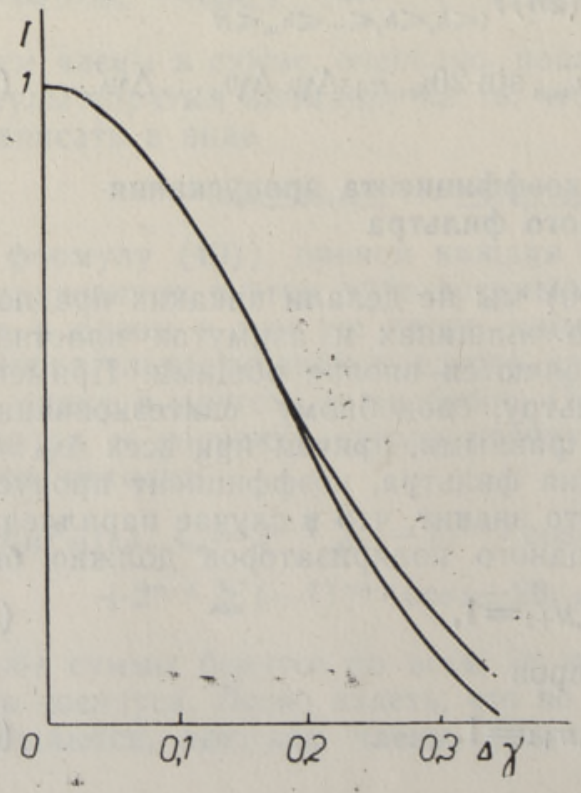

$$
F_{21} F_{21}^{*}=\cos ^{2}(3,915 \Delta \gamma)
$$

Коэффициент пропускания светофильтра по точной формуле (верхняя кривая) и по приближенной формуле (нижняя кривая). 
На рисунке показаны точная и приблнженная спектральные кривые для первого фильтра. Для второго картина сходна. Видим, что в области главного максимума пропускания получается хорошее совпадение. Наоборот, вне полосы пропускания, т. е. в области побочных максимумов, приближенная формула становится совершенно непригодной.

Другое применение формула (56) может найти при исследовании влияния наклонности падения света на фильтр. Если угол падения отличен от нуля, но мал, то толщины пластинок заменяются некоторыми эффективными значениями, мало отличающимися от первоначальных. Все $\Delta \gamma_{k}$ тогда различны. Формула (56) позволяет выполнять быструю оценку влияния этих отклонений. При этом следует, однако, учитывать также обусловленное наклонностью падения эффективное изменение азимутов.

\section{Л И Т Е Р А Т Р А}

1. К ард П., Изв. АН ЭССР, Физ. Матем., 25, № 4, 359-365 (1976).

2. К а д П., Их ер Х., Изв. АН ЭССР, Физ. Матем., 26, № 1, 13-27 (1977).

3. К а рд П. Г., Анализ и синтез многослойных интерференционных пленок, Таллин, «Валгус», 1971.

Тартуский государственный университет

Поступила в редакцию $8 / \mathrm{VI} 1981$

F. KARD

\section{INTERFERENTS-POLARISATSIOONVALGUSFILTRITE TEOORIA POHIVALEMID}

Maatriks $F$, mis teisendab interferents-polarisatioonfiltrile langeva valguse polarisatsioonimaatriksi îltri läbinud valguse polarisatsioonimaatriksiks, avaldub valemiñega

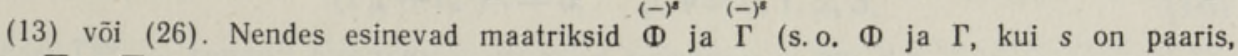
ja $\bar{\Phi}$ ja $\bar{\Gamma}$, kui $s$ on paaritu) on määratud valemitega (6)-(9), kus $\varphi$ on kaksikmurdva plaadi asimuut ja $\gamma$ on valemi (10) järgi plaadi paksusega $h$ võrdeline suurus ( $\lambda$ on lainepikkus, $n_{o}$ hariliku ja $n_{e}$ ebahariliku kiire murdumisnäitaja). Valemis (13) on $s=0,1, \ldots, N$ ( $N$ on plaatide arv) ja summa võetakse üle kõigi kombinatsioonide $k_{1}, k_{2}, \ldots, k_{s}$, kusjuures $1 \leqslant k_{1}<k_{2}<\ldots<k_{s} \leqslant N$ ja $l \neq k$. Valemis (26), kus $\varphi_{k, k+1}$ on valemitega (17) ja (19) defineeritud suurused, $x$ on kas sin vōi cos; summa võetakse üle kõigi siinuste ja koosinuste kombinatsioonide; $s=0,1, \ldots, N+1$ on siinuste arv kombinatsioonis; arvud $\varepsilon_{1}, \varepsilon_{2}, \ldots, \varepsilon_{N}$ on +1 vōi -1 nōnda, et $\left.x \varphi_{k, k+1}=\begin{array}{c}\cos \\ \sin \end{array}\right\} \varphi_{h, k+1}$ korral on $\varepsilon_{k} \varepsilon_{k+1}= \pm 1$, kus $\varepsilon_{0}=1$. Filtri läbilaskvustegurid $F_{11} F_{11}^{*}$ (sisend- ja väljundpolarisaatori paralleelorientatsiooni korral) vooi $F_{21} F_{21}^{*}$ (polarisaatorite ristuvate orientatsioonide korral) avalduvad ridadena (49) ja (50), kus $\Delta \gamma_{k}$ on määratud valemitega (33) ja (34); $\Theta_{0, N+1}$ on määratud valemiga (36), kus $N_{1}$ ja $N_{3}$ on plaatide arvud, millel $r=1$ või $r=3$, ning $m_{1}, m_{2}, \ldots, m_{N_{1}+N_{3}}$ nende plaatide indeksid; $\theta_{k t}$ on määratud valemiga (39), $\boldsymbol{\theta}_{0 k}$ ja $\boldsymbol{\theta}_{l, N+1}$ aga järgmiselt: $\boldsymbol{\theta}_{0 k}$ sisaldab kōik need $\boldsymbol{\theta}_{0, N+1}$ liikmed, mille indeks on väiksem kui $k$, ja liikme $\pm \varphi_{k} ; \boldsymbol{\theta}_{l, N+1}$ sisaldab kõik need $\Theta_{0, N+1}$ liikmed, mille indeks on suurem kui $l$, ja liikme $\pm \varphi l$; mõlema suuruse liikmete märgid vahelduvad. Kitsa läbilaskvusribaga filtri puhul summeerub $F_{11} F_{11}^{*}$ avaldis (49) ligikaudu valemiks (56); samasuguse kuju saab ka $F_{21} F_{21}^{*}$ avaldis (50), ent esimesel juhul kehtib valem (51), teisel juhul (52). Valem (56) kehtib suure täpsusega läbilaskeriba kohal (vt. joon.), ent väljaspool seda ei kehti. 


\section{BASIC THEORETICAL FORMULAE FOR THE BIREFRINGENT CHAIN LIGHT-FILTERS}

The formulae (13) and (26) are the two different forms of the matrix $F$, which transforms the polarization matrix of the light, passing a birefringent chain light-iilter. In these formulae the matrices $\Phi^{s}$ and ${ }^{(-)^{s}}$ (i. e. $\Phi$ and $\Gamma$, if $s$ is even, and $\bar{\Phi}$ and $\bar{\Gamma}$, if odd) are defined by the formulae (6)-(9), where $\varphi$ 's are the azimuths of the birefringent plates, of which the filter consists; $\gamma$ is defined by the formula $(10)$, where $h$ is the thickness of a plate, $\lambda$ is the wave-lenght, $n_{o}$ and $n_{e}$ are the refractive indices of the plates. In the formula (13) $s=0,1, \ldots, N, N$ being the number of plates, $l \neq k$, and summation runs over all the sets of the indices $k_{1}, k_{2}, \ldots, k_{s}$ with $1 \leqslant k_{1}<k_{2}<\ldots<k_{s} \leqslant N$. In the formula $(26)$ the quantities $\varphi_{k, k+1}$ are defined by the formulae (17) and (19); $\mathrm{x}$ means cosine or sine; summation runs over all the possible combinations of cosines and sines, $s=0,1, \ldots, N+1$ being the number of sines; the numbers $\varepsilon_{1}, \varepsilon_{2}, \ldots, \varepsilon_{N}$ are equal to +1 or -1 , so that $\left.\mathrm{x} \varphi_{k, k+1}=\begin{array}{c}\cos \\ \sin \end{array}\right\} \varphi_{k, k+1}$ implies $\varepsilon_{k} \varepsilon_{k+1}= \pm 1$ and $\varepsilon_{0}=1$. The transmittance $F_{11} F_{11}^{*}$ of the filter (when in- and output polarizers are parallel) or $F_{21} F_{21}^{*}$ (when polarizers are crossed) are expanded into the series (49) and (50), where $\Delta \gamma_{k}$ 's are defined by the formulae (33) and (34); $\boldsymbol{\Theta}_{0, N+1}$ is defined by the formula $(36)$, where $N_{1}$ and $N_{3}$ are the numbers of plates having $r=1$ or $r=3$, and $m_{1}, m_{2}, \ldots, m_{N_{1}+N_{3}}$ are their indices; $\theta_{0 k}$ and $\theta_{l, N+1}$ are defined as follows: $\Theta_{0 k}$ includes all terms of $\Theta_{0, N+1}$, whose indices are smaller than $k$, and the term $\pm \varphi_{k} ; \boldsymbol{\theta}_{l, N+1}$ includes all terms of $\boldsymbol{\theta}_{0, N+1}$, whose indices are greater than $l$, and the term $\pm \varphi l$; the signs of terms of both quantities are alternating. Finally, $\boldsymbol{\theta}_{k l}$ is defined by the formula (39). In the case of narrow pass-band filter, when the condition (51) holds, the expansion (49) can approximately be summed up to the formula (56). The form-identical expression holds also for $F_{21} F_{21}{ }^{*}$, but instead of (51) the condition (52) must be used. The approximation (56) fits quite well inside the pass-band (s. fig.), but outside the pass-band is totally wrong. 